\title{
ACQUIRED PALSY OF THE OCULOMOTOR, TROCHLEAR AND ABDUCENS NERVES
}

\author{
P. A. C. TIFFIN ${ }^{1}$, C. J. MacEWEN ${ }^{1}$, E. A. CRAIG ${ }^{1}$ and G. CLAYTON ${ }^{2}$ \\ Dundee
}

\section{SUMMARY}

There have been few studies primarily concerned with the relative frequencies, aetiologies and prognoses of ocular motor palsies. Those published have emanated largely from neurological tertiary referral centres rather than primary ophthalmology departments. We have performed a retrospective study of all patients with acquired III, IV or VI cranial nerve palsy who were seen in the orthoptic department at Ninewells Hospital, Dundee, over the 9 year period from 1984 to 1992. A total of 165 cases were identified. VI nerve palsies accounted for the majority of patients $(57 \%)$, with IV nerve palsies $(21 \%)$ occurring more frequently than III nerve palsies (17\%) and multiple palsies (5\%). Thirtyfive per cent of cases were of unknown aetiology and $32 \%$ of vascular aetiology. The incidence of sinister pathology - neoplasia (2\%) and aneurysm (1\%) - was surprisingly low. Fifty-seven per cent of all patients made a total recovery (in a median time of 3 months) and $80 \%$ made at least a partial recovery. The results are contrasted with those of previous studies and the value of associated symptoms and of further investigation in the assessment of these patients is discussed.

Acquired palsies of cranial nerves III, IV or VI are commonly initially referred to the ophthalmology emergency department and are often perceived as a sign of serious underlying pathology.

There have been few studies primarily concerned with the relative incidence, aetiology and prognosis of extraocular muscle palsy. Those published largely relate to cohorts of patients attending the Mayo Clinic from the 1950 s to $1988 .^{1-4}$ The figures obtained from this large tertiary referral centre have shown a relatively high incidence of isolated oculomotor cranial nerve palsy - occurring less frequently than abducens nerve palsy but more

From: Departments of ${ }^{1}$ Ophthalmology and ${ }^{2}$ Orthoptics, Ninewells Hospital and Medical School, Dundee, UK.

Correspondence to: Dr P. Tiffin, Department of Ophthalmology, Ninewells Hospital and Medical School, Dundee DD1 9SY, UK. commonly than trochlear nerve palsy. In addition, although the major cause of ocular palsy was consistently classified as undetermined $(25 \%)$, a relatively high proportion of cases were recorded as secondary to sinister pathology: neoplasia $(18 \%)$ and aneurysm $(7 \%)$.

There have been several studies relating to series of patients seen in the more general setting of both orthoptic and ophthalmic clinics. ${ }^{5-9}$ However, these have concentrated on groups of patients presenting with the somewhat broader diagnosis of diplopia rather than only those with acquired nerve palsies per se. Acquired ocular motor nerve palsy accounted for $40-60 \%$ of patients presenting with binocular diplopia. Although abducens nerve palsy occurred most frequently in most of these reports, ${ }^{6,8,9}$ oculomotor nerve palsy was found most often in one ${ }^{5}$ and occurred more frequently than trochlear palsy in another. ${ }^{8}$ Overall, however, these series have pointed towards a more benign condition, with one study reporting on underlying rate of neoplasia of only $6.8 \%,{ }^{7}$ and others an unknown or presumed vascular aetiology in over $50 \%$ of cases. ${ }^{8,9}$ In addition a high rate of spontaneous recovery of up to $70-78 \%$, generally occurring within the first 3 months, was reported. 8,10

We therefore performed a retrospective review of all patients with acquired III, IV or VI cranial nerve palsies (either isolated or in combination) who were seen in the orthoptic department at Ninewells Hospital, Dundee over the 9 year period 1984 to 1992. In so doing we hoped to develop a more accurate picture of the relative frequencies, aetiologies and prognoses of ocular palsies as seen in a typical general ophthalmology department in the UK.

\section{PATIENTS AND METHODS}

All orthoptic records for the period January 1984 to December 1992 were reviewed. The hospital records of patients who, from these orthoptic notes,

Eye (1996) 10, 377-384 C 1996 Royal College of Ophthalmologists 
appeared to have presented with a III, IV or VI cranial nerve palsy were examined in detail. The diagnosis was confirmed on the basis of the documented history and examination including Hess charts. All cases, whether single or multiple palsies, and those occurring in combination with other ophthalmic or neurological signs, were included. Cases of III nerve palsy were further classified as total or partial depending on the extent to which the extraocular muscles were affected, and independently of pupillary involvement.

Details of the patient's age, sex and previous medical history, with particular regard to hypertension, vascular disease and diabetes, were recorded. In addition, the time from onset of symptoms to presentation was noted, together with any relevant history of trauma. Documented pain, pupillary involvement and the presence of other related ophthalmic or neurological signs were recorded. Similarly, any investigations performed, particularly neuroradiological assessment, and their results were noted. The presence of subsequent recovery, whether total or partial, and the length of follow-up or time from onset to total recovery was also examined. Total recovery was taken as having occurred when the extraocular movements had recovered and there was no longer any diplopia or requirement for prismatic correction. Patients who had been seen with a previous episode of an ocular motor palsy, or who subsequently developed one or more further episodes, were also noted.

Cases were also classified on the basis of aetiology as: unknown, vascular, neoplastic, aneurysm, traumatic or other. As with previous studies those classified as vascular were done so speculatively on the basis of pre-existing or newly diagnosed diabetes, hypertension, ischaemic heart disease or peripheral vascular disease in the absence of other neurological signs.

\section{RESULTS}

A total of 316 possible cases were identified from the orthoptic department records. For 247 (78\%) of these the hospital records were traced. One hundred and seventy-four patients were diagnosed as having had either III, IV or VI nerve palsies. Seventy-three patients were excluded on the basis of an established alternative diagnosis (e.g. dysthryoid eye disease, blowout fracture, myasthenia, skew deviation, decompensated phorias) or because follow-up occurred elsewhere. A further 9 patients with congenital palsies (8 IV nerve, 1 III nerve) were also excluded in order to limit the study to cases of acquired palsy and to facilitate comparison with previous studies.

The patient group therefore comprised 165 patients (Table I), 77 of whom were male and 88
Table I. Distribution of patients by age, sex and laterality for each nerve palsy $(n=165)$

\begin{tabular}{lcccc}
\hline & \multicolumn{4}{c}{ Nerve palsy } \\
\cline { 2 - 5 } & VI & IV & III & Multiple \\
\hline$n$ & 93 & 35 & 28 & 9 \\
$(\%)$ & $(57)$ & $(21)$ & $(17)$ & $(5)$ \\
Mean age (years) & 62 & 59 & 67 & 60 \\
Age range (years) & $1-91$ & $8-83$ & $3-84$ & $30-71$ \\
Male & 39 & 21 & 11 & 6 \\
Female & 54 & 14 & 17 & 3 \\
Right & 33 & 16 & 15 & 3 \\
Left & 49 & 18 & 13 & 6 \\
Bilateral & 11 & 1 & 0 & 0 \\
\hline
\end{tabular}

female. Age range was 1-91 years (mean 62.5 years, SD 17.5 years).

Table I gives the relative frequencies of each nerve palsy, and distributions by age, sex and laterality. It can be seen that VI nerve palsy accounted for nearly $60 \%$ of cases and IV nerve palsy occurred next most frequently, ahead of III nerve palsies. Multiple palsies occurred least frequently. There were 12 bilateral cases (11 VI nerve and 1 IV nerve).

Tables II and III show the aetiologies of palsies for the patient group as a whole. For comparison the cumulative results of the four Mayo Clinic series given by Richards et al. ${ }^{4}$ are shown. It can be seen that more than two-thirds of the cases in our study had an unknown or a vascular cause. There were only 4 cases secondary to neoplasia (1 VI nerve, 2 III nerve and 1 multiple) and 2 associated with intracranial aneurysm (1 VI nerve and 1 multiple). Eighteen patients $(11 \%)$ were diabetic and this proportion was remarkably consistent across each kind of palsy.

The frequency of recovery across the whole patient group was high, $57 \%$ of all patients making a total recovery and $80 \%$ making at least a partial recovery. Recovery occurred most frequently in patients with unknown or vascular aetiologies $(84 \%$ and $85 \%$ respectively made at least partial recovery), but appeared less frequent for those with trauma $(46 \%)$, neoplasia $(25 \%)$ and aneurysm $(50 \%)$. With the exception of multiple palsies the frequency and speed of total recovery were remarkably constant across different nerve palsies.

\section{VI (Abducens) Nerve Palsy}

\section{Aetiology}

Table II shows the frequency of different aetiologies for VI nerve palsy. The majority were of unknown or vascular origin - accounting for $33 \%$ each. There was only 1 case attributable to neoplasia, in which a VI nerve palsy developed following radiotherapy for a nasopharyngeal carcinoma. Only 1 case secondary to aneurysm occurred: This patient had a painful partial VI nerve palsy, symptomatic for 18 months, secondary to an intracavernous aneurysm. In both cases there was subsequent progression to involvement of the ipsilateral III nerve. 
Table II. Aetiology of all nerve palsies $(n=165)$

\begin{tabular}{|c|c|c|c|c|c|c|c|c|c|c|c|}
\hline \multirow[b]{3}{*}{ Aetiology } & \multicolumn{10}{|c|}{ Nerve palsy } & \multirow{3}{*}{$\begin{array}{c}\text { Richards et al. } \\
(\%)\end{array}$} \\
\hline & \multicolumn{2}{|c|}{ VI } & \multicolumn{2}{|c|}{ IV } & \multicolumn{2}{|c|}{ III } & \multicolumn{2}{|c|}{ Multiple } & \multicolumn{2}{|c|}{ All } & \\
\hline & $n$ & $(\%)$ & $n$ & $(\%)$ & $n$ & $(\%)$ & & $(\%)$ & $n$ & $(\%)$ & \\
\hline Unknown & 31 & (33) & 16 & (46) & 9 & $(32)$ & 1 & (11) & 57 & (35) & 25 \\
\hline Vascular & 31 & (33) & 9 & (26) & 13 & (46) & 0 & (0) & 53 & (32) & 15 \\
\hline Trauma & 3 & (3) & 9 & (26) & 0 & $(0)$ & 1 & (11) & 13 & (8) & 16 \\
\hline Neoplasia & 1 & (1) & 0 & (0) & 2 & (7) & 1 & (11) & 4 & (2) & 18 \\
\hline Aneurysm & 1 & (1) & 0 & (0) & 0 & $(0)$ & 1 & (11) & 2 & (1) & 7 \\
\hline Others & 26 & (28) & 1 & (3) & 4 & (14) & 5 & (55) & 36 & $(22)$ & 18 \\
\hline Total & 93 & $(100)$ & 35 & $(100)$ & 28 & $(100)$ & 9 & (100) & & & \\
\hline Diabetic & 10 & (11) & 4 & (11) & 3 & (11) & 1 & (11) & 18 & (11) & 13 \\
\hline
\end{tabular}

\section{Recovery}

Fifty-six patients $(61 \%)$ made a complete recovery, the median time for which was 3 months (range 1-58 months). Twenty-six patients $(26 \%)$ made only a partial recovery and $12(13 \%)$ made none. For those cases of unknown or vascular aetiology the rate of total recovery increased to $70 \%$, with $92 \%$ making at least a partial recovery. Conversely in the remaining patients only $48 \%$ made a total recovery and $25 \%$ made no recovery.

\section{Pain}

Fifteen patients were recorded as complaining of pain at presentation. Although pain was associated with serious pathology in 3 cases, in each of these there were other signs and symptoms suggestive of this. Eight cases with pain were of vascular or unknown aetiology. The incidence and rate of recovery in patients with pain were also similar to the rest of the VI nerve palsy group.

\section{Young Patients}

There were 24 patients aged $<55$ years. Three patients had long-standing palsies of more than 6 months' duration and already had established aetiologies; 21 patients therefore presented fairly acutely. Fourteen of 21 had neuro-imaging. Eleven cases $(52 \%)$ were of unknown/vascular aetiology, with a further 2 having a presumed post-viral inflammatory cause; 5 patients had multiple sclerosis (MS; 3 had pre-existing diagnoses and 2 were diagnosed follow-

Table III. 'Other' aetiologies of all nerve palsies $(n=36)$

\begin{tabular}{lccccc}
\hline & \multicolumn{5}{c}{ Nerve palsy } \\
\cline { 2 - 6 } Aetiology & VI & IV & III & Multiple & Total \\
\hline HZO & 4 & 0 & 2 & 2 & 8 \\
MS & 5 & 0 & 0 & 0 & 5 \\
Brainstem CVA & 3 & 0 & 1 & 2 & 6 \\
Temporal arteritis & 3 & 0 & 0 & 0 & 3 \\
Post-surgical & 2 & 1 & 0 & 0 & 3 \\
? Post-viral & 3 & 0 & 0 & 0 & 3 \\
C-C fistula & 1 & 0 & 0 & 1 & 2 \\
Miscellaneous & 5 & 0 & 1 & 0 & 6 \\
Total & 26 & 1 & 4 & 5 & 36 \\
\hline
\end{tabular}

HZO, herpes zoster ophthalmicus; MS, multiple sclerosis; Brainstem CVA, brainstem cerebrovascular accident; C-C fistula, carotico-cavernous fistula. ing subsequent referral for neurological investigation on the basis of other symptoms and signs). The recovery rate in young patients was similar to that of the whole group, with 14 of $21(66 \%)$ making a total recovery and only one making no recovery at all.

\section{Bilateral Cases}

Eleven patients had bilateral VI nerve palsies. These patients appeared slightly younger (mean age 50 years), with 6 being 40 years or less. In 4 of the cases there was an obvious pre-existing diagnosis (1 trauma, 1 known carcinomatosis, and 2 patients with MS). Surprisingly, of the 7 patients remaining a definitive diagnosis was made in only 1 case (brainstem haematoma), despite 6 cases having had a CT scan and 2 angiography. Five of the 11 patients made a total recovery and 3 made none.

\section{Investigations}

CT scans were obtained in 33 cases $(35 \%)$ of VI nerve palsy. The 'diagnostic yield' was rather poor, providing a positive diagnosis in only the cases of intracavernous aneurysm and brainstem haematoma. In 10 of the cases scanned there were no 'atypical' features suggestive of any likely identifiable underlying aetiology. Cerebral angiography was performed in 5 patients. Only 3 patients had MRI scans. Other routine investigations revealed little previously undiagnosed pathology. Two patients were diagnosed as diabetic and 1 as hypertensive; 3 patients were diagnosed as probably having temporal arteritis on the basis of elevated erythrocyte sedimentation rates $(110,128$ and $70 \mathrm{~mm} / \mathrm{h})$, although 2 of these subsequently had negative temporal artery biopsies.

Table IV. Aetiologies of III nerve palsy $(n=28)$

\begin{tabular}{lcccc}
\hline Aetiology & $\begin{array}{c}\text { Total } \\
(n)\end{array}$ & $\begin{array}{c}\text { Partial } \\
(n)\end{array}$ & $\begin{array}{c}\text { All } \\
(\%)\end{array}$ & $\begin{array}{c}\text { Richards } \text { et al. } \\
(\%)\end{array}$ \\
\hline Unknown & 2 & 7 & 32 & 24 \\
Vascular & 5 & 8 & 46 & 20 \\
Trauma & 0 & 0 & 0 & 15 \\
Neoplasm & 0 & 2 & 7 & 12 \\
Aneurysm & 0 & 0 & 0 & 16 \\
Other & 3 & 1 & 14 & \\
Total & 10 & 18 & 99 & \\
Diabetic & 2 & 1 & 11 & 6 \\
\hline
\end{tabular}




\section{IV (Trochlear) Nerve Palsy}

IV nerve palsy was the second most frequent ocular palsy, although it occurred approximately 3 times less frequently than VI nerve palsy (Table I). IV nerve palsy occurred more commonly in males $(2: 1)$, and at a slightly lower mean age than for other ocular motor palsies.

\section{Aetiology and Recovery}

Table II shows the frequency of different aetiologies for IV nerve palsy. Nearly half were of unknown cause, with vascular and trauma accounting for $26 \%$ each. There were no instances of malignancy or aneurysm. Seventeen of the 34 patients $(50 \%)$ made a total recovery. The median time to total recovery was again 3 months, the longest time being 8 months. Eight made only partial recovery and $9(26 \%)$ made none. Only 3 of the 9 cases due to trauma made a complete recovery.

\section{Pain}

Only 3 cases were associated with pain. All had normal scans (2 CT, $1 \mathrm{MRI}$ ) and were of unknown aetiology. All 3 made a total recovery with no sequelae.

\section{Bilateral Cases}

There was only 1 documented case of bilateral IV nerve palsy, seen 6 months following closed head injury sustained in a road accident.

\section{Investigations}

Six patients had CT scans and 1 had an MRI scan. No abnormalities were reported. No patient had cerebral angiography.

\section{III (Oculomotor) Nerve Palsy}

Aetiology

Table II shows the frequency of different aetiologies for all III nerve palsies. Interestingly there were no cases of isolated III nerve palsy secondary to intracranial aneurysm. There were 2 cases secondary to neoplasia. The first presented with a painful, pupilsparing partial III palsy associated with ipsilateral proptosis, disc swelling, and reduced maxillary sensation caused by an ethmoidal tumour. The second case also presented with a painful, pupilsparing partial III nerve palsy secondary to a III nerve schwannoma.

\section{Total Paresis}

Ten patients presented with total paresis of all extraocular muscles innervated by the oculomotor nerve (Table IV). Seven of these were of unknown/ vascular aetiology. Of the 3 others, 2 were due to zoster, and the remaining case was secondary to a brainstem infarction which was associated with other localising neurological signs. Four of the 10 patients had pupillary involvement and 2 had pain; only the patient with a brainstem haematoma had both.

\section{Partial Paresis}

Eighteen patients presented with partial III nerve palsy. The majority of cases $(15 / 18,83 \%)$ were again of unknown/vascular aetiology. However, both cases secondary to neoplasia occurred in this group. The remaining case was thought to be due to arteritis on the basis of the angiographic features. Five of the 18 cases showed pupil involvement and 6 had pain; 2 patients had both.

\section{Recovery}

Patients presenting wtih total III nerve palsy had a high rate of recovery, 8 of $10(80 \%)$ making a total recovery in a median time of 3 months (longest time to recovery was 6 months). Only 1 patient with a total palsy showed no sign of recovery, but had pupillary signs of aberrant regeneration at presentation and had had symptoms for 5 years. Recovery appeared less consistent in partial III nerve palsy, with only 10 patients $(56 \%)$ making a complete recovery in a median time of 2 months (maximum 24 months). Two patients with partial palsy made only a partial recovery and $6(33 \%)$ made none.

\section{Investigations}

Three patients with total III nerve palsy had CT sans and 1 proceeded straight to angiography. Seven patients with partial palsy had CT scans, 2 had angiography in addition, and 1 also had an MRI scan.

Table V. Multiple cranial nerve palsies $(n=9)$

\begin{tabular}{lcllll}
\hline Palsies & Age (years) & Other symptoms/signs & Investigations & Aetiology & Recovery \\
\hline III + IV & 30 & Hemiparesis & CT + MRI & Brainstem CVA & None \\
IV + VI & 69 & Zoster & - & HZO & Total 2 months \\
III + VI & 61 & Nystagmus, oscillopsia & CT & ?? Metastasis & None \\
III + VI & 69 & Oesophageal carcinoma & - & Metastasis & No follow-up \\
III + VI & 61 & Pain & CT & Intravenous aneurysm & None \\
III + VI & 66 & Pain & CT + Angiography & None \\
III + VI & 58 & Symptoms since road accident & - & HZO & Total 3 months \\
III + VI & 71 & Zoster previously & - & C-C fistula & None \\
III + VI & 58 & Pain & CT + Angiography & & \\
\hline
\end{tabular}

HZO, Herpes zoster ophthalmicus; MS, multiple sclerosis; Brainstem CVA, brainstem cerebrovascular accident; C-C fistula, caroticocavernous fistula. 


\section{Multiple Nerve Palsies}

Multiple nerve palsies occurred least frequently (Table I). Table V shows details of presentation, investigation, aetiology and recovery for individual patients. It can be seen that a definitive cause was found for every patient except one. This patient had terminal oesophageal carcinoma and was not investigated or subsequently reviewed. Recovery rates were poor, with only the 2 zoster patients showing recovery; total recovery occurred by 2 and 3 months respectively.

\section{DISCUSSION}

We have performed a retrospective review of cases of acquired ocular palsy seen in the setting of a typical general ophthalmology clinic in the UK. Although the numbers of patients in the groups, other than that for VI nerve palsy, are not particularly large, this study nevertheless represents data accumulated over a 9 year period at a hospital with a catchment population of around 200000 .

One criticism of this retrospective study may be that only 247 case notes out of 316 possible cases identified were obtainable. However, this still represents a retrieval rate of $78 \%$. Furthermore it would appear unlikely to have significantly distorted our results since the case notes which could not be traced were not biased towards any particular patient group (e.g. those who had since died) or to those with any particular nerve palsy.

In tracing only those patients who were seen in the orthoptic department, we may also have underestimated the number with serious pathology. Such patients may have been referred on to other hospital departments without first having had an orthoptic assessment. This seems unlikely to have occurred to any great extent for two reasons. Firstly the eye clinic, eye casualty and orthoptic department share a common outpatient area, and most patients with diplopia are therefore assessed in the orthoptic clinic as well as being seen by a doctor. Secondly, during the period of this study the vast majority of intracranial aneurysms in Dundee were operated on by one neurosurgeon. Detailed operating records including a presenting history were available for 6 of the 9 years of our study period. Cross-checking against these records we found no additional cases of aneurysm which had been seen initially in the eye department. Only 1 further case of neoplasia - an orbital lymphoma causing multiple ocular palsies was found.

Our data confirm the impression that VI nerve palsy : occurs far more commonly than other ocular palsies, accounting for $57 \%$ of cases. However, in contrast to some previous studies, ${ }^{1-5,8,11}$ IV nerve palsy was seen more often than III nerve palsv - even allowing for exclusion of those cases thought to be congenital in origin.

Similarly, the relative frequencies of cases of unknown aetiology, and to a greater extent so-called vascular aetiology, were greater than previously described in some series. ${ }^{1-4,11}$ In this study $35 \%$ of all cases were of unknown aetiology and $33 \%$ were assigned a vascular cause. The corresponding figures for the study by Richards et al. ${ }^{4}$ were only $25 \%$ and $15 \%$, which reflects the different patient populations for the two clinical settings. In contrast those studies arising from ophthalmic and orthoptic clinics have produced findings similar to ours, with no aetiology identified in approximately $40 \%$ of cases in two studies $^{5,8}$ and a vascular aetiology presumed in $40 \%$ of cases in another. ${ }^{9}$ The classification of any palsy as vascular in aetiology is of course purely speculative and based merely on the presence of existing vasculopathy or risk factors and the lack of any other neurological signs. Contrary to previous studies, we did not find evidence of a lower rate of recovery in patients whose palsy had an unknown as compared with a vascular cause, ${ }^{4}$ the rates of at least partial recovery being $84 \%$ and $85 \%$ respectively. These recovery rates were virtually identical across each kind of nerve palsy. In addition the time to total recovery was similar for all cases of unknown and vascular aetiology regardless of the cranial nerve involved. It seems likely, therefore, that the underlying pathological mechanism was similar in the two groups, making differentiation between the two even more arbitrary.

The incidence of underlying neoplasia and, to a greater extent aneurysm, was surprisingly low. Previous series have reported an incidence of neoplasia between $6 \%$ and $26 \% .{ }^{1-4,7,11}$ In our study there were only 4 cases secondary to tumour $(2 \%)$. Of these 1 had been diagnosed prior to the onset of VI nerve palsy, 1 presented with multiple palsies (III and VI) and 1 developed a III nerve palsy associated with proptosis and other signs. Only 1 case of neoplasia therefore presented with a purely isolated nerve palsy (a painful III).

Similarly the rate of aneurysm reported in previous, more neurologically based series was between $4 \%$ and $11 \% .^{1-4,11} \mathrm{We}$ encountered only 2 cases of aneurysm (1\%), both of which were intracavernous. One presented with multiple palsies (III and VI) and the other, though isolated, had been symptomatic for 18 months. Nolan ${ }^{5}$ noted that of 6 aneurysms found in 121 patients with ocular palsies $(5 \%)$, all but 1 presented with multiple nerve involvement. Trimble and Kelly ${ }^{8}$ reported only 2 cases of aneurysm in 94 patients presenting with diplopia and Morris ${ }^{9}$ reported no aneurysms in 206 patients with binocular diplopia. Taken in conjunction with our results it wnuld therefore annear thet 
acute ocular palsy due to underlying aneurysm presents to the eye clinic more rarely than hitherto generally believed.

The frequency of recovery across the whole patient group was high, with $80 \%$ of all patients making at least partial recovery. Recovery occurred most frequently in patients whose palsies had an unknown or vascular aetiology, and less frequently in those whose palsy was caused by trauma, neoplasia and aneurysm - although patient numbers were small for these latter groups. Similar results have been reported in previous series. ${ }^{4-6,8,10}$ With the exception of multiple palsies the frequency and speed of total recovery were remarkably constant across different nerve palsies. Between $50 \%$ and $60 \%$ of each nerve palsy group made a total recovery, for which the median time taken was consistently 3 months, regardless of the nerve involved. Within the III nerve palsy group there was a higher rate of total recovery for patients with total palsy $(80 \%)$ than for those with partial palsy (55\%).

With respect to VI nerve palsy several points are worthy of note. As seen in the context of an eye clinic, VI nerve palsy appears a relatively 'benign' disease. Two-thirds of patients had a palsy of unknown/vascular aetiology, and of these $70 \%$ made a total recovery. Although the aetiologies encountered were quite diverse, 'serious' underlying pathology in the absence of an atypical history and examination appears rare.

Pain did not appear a particularly useful diagnostic factor in VI nerve palsy. Despite a relatively higher rate of investigation in patients with pain, over half of these cases were still of unknown/vascular aetiology, and those patients with serious pathology had other symptoms and signs. Furthermore the prognosis for recovery was similar to the rest of the VI nerve palsy group.

Younger patients are also often viewed with some concern. However, again in the absence of other atypical features, the underlying aetiologies in patients under 55 years old, with the exception perhaps of those with MS, were similar to the whole VI nerve palsy group. Two MS patients (aged 32 and 54 years) were newly diagnosed following VI nerve palsy, although both had other neurological symptoms. The prognosis for recovery in young patients was also similar to that for the rest of the VI nerve palsy group.

Similarly all cases of bilateral VI nerve palsy occurring in patients without a pre-existing diagnosis or other signs were benign, despite a high rate of investigation. The incidence and speed of recovery were again similar to the rest of the group.

Eight cases of IV nerve palsy thought to be congenital were excluded from the study. IV nerve palsies were diagnosed as congenital where there were well-developed muscle sequelae and concomitance on the Hess chart, an increased vertical fusion range and no evidence of torsional diplopia. These patients were generally in their second or third decades and often gave a history of intermittent diplopia and symptoms of decompensation. The rate of congenital IV nerve palsies $(8 / 43=19 \%)$, although higher than the $12 \%$ reported by Richards et al. ${ }^{4}$ is lower than that reported by others (Morris ${ }^{9} 54 \%$ ). Since we were primarily concerned with patients with only acquired palsies we did not retrieve case notes for patients who from the orthoptic records appeared to have a congenital palsy. Our study therefore probably underestimates the true incidence of congenital IV nerve palsy.

IV nerve palsy occurred more commonly in males and at a slightly lower mean age. This is probably a reflection of the higher incidence of underlying traumatic aetiology. There were no cases secondary to neoplasia or aneurysm. The rarity of these aetiologies in isolated IV nerve palsy has been previously noted. ${ }^{4}$ Only 1 bilateral case was documented, although bilateral involvement may easily have been overlooked in some of the cases recorded as unilateral. Given the relatively high incidence of trauma in this group, it is disconcerting that only 3 of 9 traumatic cases made a spontaneous total recovery. Again the presence of pain did not appear to correlate with more serious pathology. All 3 patients with a painful IV nerve palsy had normal CT scans and subsequently made a complete recovery.

The III nerve palsy group was remarkable for the fact that not one case secondary to aneurysm was found. Previous studies have recorded underlying aneurysm in $16-29 \%$ of patients with III nerve palsy. ${ }^{1-4,11,12}$ Conversely the incidence of vascular cases $(46 \%)$ and, to a lesser extent, those with unknown aetiology (32\%), were much higher than in these studies.

The possibility of underlying aneurysm in III nerve palsy with pupillary involvement has been well documented. ${ }^{2,411-13}$ However, the possibility of a pupil-sparing III nerve palsy with only partial extraocular muscle involvement also being due to aneurysmal compression has been highlighted previously. ${ }^{14}$ Both our cases of tumour presented in this manner. Pupil involvement has been documented in up to $95 \%$ of cases of III nerve palsy due to aneurysm but only $60-80 \%$ of vascular cases. ${ }^{2,12}$ Our group contained 9 patients $(32 \%)$ who presented with pupil involvement ( 3 with pain) yet none was found to have an aneurysm. Although 4 of 8 patients with pain had a palsy of unknown or vascular aetiology, both cases of neoplasia and the case of angiographically diagnosed vasculitis presented with pain.

Routine investigation of a patient with an ocular 
motor palsy has traditionally included a skull radiograph, full blood count, erythrocyte sedimentation rate (ESR) or plasma viscosity, and blood glucose. Although 26 patients in our study had a skull radiograph performed, none was diagnostically helpful, and in our opinion this investigation is no longer merited. ${ }^{15}$ Similarly no patient showed any abnormality on routine full blood count other than a mild lymphocytosis in 1 patient following a recent virallike illness. A full blood count therefore also seems of questionable value. Blood glucose testing revealed only 2 previously undiagnosed diabetics. Given the incidence of diabetes in this age group this does not appear remarkable. The ESR was elevated sufficiently to suggest temporal arteritis in 3 patients, although 2 subsequently had normal temporal artery biopsies.

From our experience immediate CT scanning of patients with isolated IV or VI nerve palsy, and no atypical features in the presenting history and examination, is unlikely to be abnormal and is therefore unnecessary. Probably the major dilemma faced when assessing a patient with III nerve palsy is the need to proceed to conventional angiography in order to exclude underlying aneurysm. Routine CT and MRI scanning are probably only about $50 \%$ sensitive for detection of aneurysm, ${ }^{16}$ and conventional angiography is associated with definite risk in elderly vasculopathic individuals. The issue has been elegantly discussed previously ${ }^{13,14}$ and our relatively small series precludes any definitive conclusions. However, given the relatively small number of patients presenting with III nerve palsy and the well-documented underlying possible aetiologies, it would still seem reasonable to perform routine CT or MRI scanning in all cases. An exception to this may be in total, pupil-sparing palsy (whether painful or not), especially if occurring in a known diabetic. Progression to conventional angiography following negative scanning still appears justified in all cases of acute palsy with pupil involvement unless there are other signs suggestive of a specific aetiology. Partial palsies should be viewed with suspicion and initially monitored closely for signs of progression - unless associated with pain or occurring in a younger or 'non-vascular' patient, when early angiography would be more appropriate. MRI became available in the later stages of the study and was therefore performed on only 7 patients. More recently MR angiography has also been developed. Initial studies with MR angiography have claimed to detect aneurysms of $4 \mathrm{~mm}$ or more with a sensitivity of up to $95 \%$ in patients previously investigated with conventional arterial angiography. ${ }^{17}$ The clinical value of MRI and MR angiography in the detection of aneurysm in patients with isolated oculomotor palsy is, however, yet to be defined.
Also of interest were the $17(10 \%)$ patients who had recurrent episodes of ocular palsy. Eleven occurred in the same nerve on each occasion and involved only the VI cranial nerve. There were 3 cases of particular note. All 3 patients initially appeared to have had a benign palsy which made a complete recovery; however, all were subsequently found to have a more serious aetiology at recurrence. The first patient presented with a left VI nerve palsy which made a total recovery over 5 months. Both CT scan and cerebral angiography were negative. Four years later she developed a further left VI nerve palsy and a repeat CT scan showed a Rathkes pouch cyst. The second patient initially presented with a partial right III nerve palsy; no investigations were performed and she made a total recovery over 7 months. She was discharged but returned a year later with a recurrent right III nerve palsy, when CT scan showed a large meningioma. The third patient presented with a VI nerve palsy which made a total recovery over 3 months; a CT scan was normal. Three years later she developed combined left III and VI palsies, and a repeat CT scan revealed a giant intracavernous aneurysm which was confirmed on angiography. Such remission of ocular palsy in association with underlying skull base tumours has been previously highlighted in cases of VI nerve palsy. 18

There seems little doubt that the patients described in our study, set in the context of a general ophthalmology department, represent a different population from those described in previous, more neurological based series. ${ }^{1-4,11,12}$ Our results are more comparable with some of those relating to patients presenting to orthoptic and ophthalmic clinics with diplopia, ${ }^{5-10}$ and point to a more benign disease spectrum. Despite the advent of sophisticated neuroimaging, the majority of cases remain of unknown or presumed vascular aetiology. Serious underlying pathology in the context of an isolated ocular motor palsy presenting acutely is probably rarer than previously held. Investigations in such patients are therefore generally unlikely to be rewarding, although negative investigations in some cases do provide necessary reassurance for both patient and doctor. Patients with III nerve palsy, especially if partial or with pupil involvement, and patients with recurrent palsies of no obvious cause, even if previously investigated, should be viewed more critically. Failure to show spontaneous improvement over the first few weeks is unusual in cases with a 'benign' aetiology and may be taken as an indication for further investigation. A meticulous history and clinical examination remain the most discriminating tools available in the assessment of these patients, and allow for the most appropriate use of subsequent investigations. 
Key words: Abducens nerve, Aetiology, Oculomotor nerve, Palsy, Prognosis, Trochlear nerve.

\section{REFERENCES}

1. Rucker CW. Paralysis of the third, fourth and sixth cranial nerves. Am J Ophthalmol 1958;46:787-94.

2. Rucker CW. The causes of paralysis of the third, fourth and sixth cranial nerves. Am J Ophthalmol 1966;61:1293-8.

3. Rush JA, Younge BR. Paralysis of cranial nerves III, IV and VI: cause and prognosis in 1000 cases. Arch Ophthalmol 1981;99:76-9.

4. Richards BW, Jones FR, Younge BR. Causes and prognosis in 4278 cases of paralysis of the oculomotor, trochlear and abducens cranial nerves. Am J Ophthalmol 1992;113:489-96.

5. Nolan J. Diplopia. Br J Ophthalmol 1968;52:166-71.

6. Yapp JMS. Diplopia as a presenting symptom. $\mathrm{Br}$ Orthopt J 1973;30:52-65.

7. Trimble RB. Diplopia as a presenting sign of neoplasia. Trans Ophthalmol Soc UK 1980;100:498-500.

8. Trimble RB, Kelly V. Diplopia as a presenting symptom: a prospective study. Proc IV Int Orthopt Congr, Berne, 1979:91-4.

9. Morris RJ. Double vision as a presenting symptom in an ophthalmic casualty department. Eye 1991;5:124-9.
10. King AJ, Stacey E, Stephenson G, et al. Spontaneous recovery rates for unilateral sixth nerve palsies. Eye 1995;9:476-8.

11. Berlit P. Isolated and combined pareses of cranial nerves III, IV and VI: a retrospective study of 412 patients. J Neurol Sci 1991;103:10-5.

12. Green WR, Hackett ER, Schlezinger NS. Neuroophthalmologic evaluation of oculomotor nerve paralysis. Arch Ophthalmol 1964;72:154-67.

13. Kwan ESK, Laucella M, Hedges TR, et al. A cliniconeuroradiologic approach to third cranial nerve palsies. AJNR 1987;8:459-67.

14. Trobe JD, Arbor A. Third nerve palsy and the pupil: footnotes to the rule [editorial]. Arch Ophthalmol 1988;106:601-2.

15. Sanders R, MacEwen CJ, McCulloch A. The value of skull radiography in ophthalmology. Acta Radiol 1994;35:1-5.

16. Earnest F, Forbes G, Sandok BA, et al. Complications of cerebral angiography: prospective assessment of risk. AJNR 1983;4:1191-7.

17. Ross JS, Masaryk TJ, Modic MT, et al. Intracranial aneurysms: evaluation by MR angiography. AJNR 1990;11:449-55.

18. Volpe NJ, Lessell S. Remitting sixth nerve palsy in skull base tumours. Arch Ophthalmol 1993;111:1391-5. 